\title{
Application of fluorescein sodium in breast cancer brain-metastasis surgery
}

This article was published in the following Dove Press journal:

Cancer Management and Research

\begin{abstract}
Shi-yin Xiao'
Ji Zhang ${ }^{2, *}$

Zheng-quan Zhu ${ }^{3, *}$

You-ping $\mathrm{Li}^{4, *}$

Wei-ying Zhong ${ }^{5}$

Jian-bin Chen ${ }^{6}$

Zhen-yu $\operatorname{Pan}^{7}$

Hai-chen $\mathrm{Xia}^{3}$

'Department of Neurosurgery, Second Affiliated Hospital of Guangzhou Medical University, Guangzhou 510260, China; ${ }^{2}$ Department of Neurosurgery, State Key Laboratory of Oncology in South China, Sun Yat-sen University Cancer Center, Collaborative Innovation Center for Cancer Medicine, Guangzhou 510060 China; ${ }^{3}$ Department of Neurosurgery, Tumor Hospital Affiliated of Xinjiang Medical University, Xinshi District, Urumqi, Xinjiang 8300 II, China; ${ }^{4}$ Department of Neurosurgery, First Affiliated Hospital of Nanchang University, Nanchang, Jiangxi 330046, China; ${ }^{5}$ Department of Neurosurgery, Qilu Hospital, Shandong University, Jinan 2500 I2, China; ${ }^{6}$ Department of Neurosurgery, Tongji Hospital, Tongji Medical College, Huazhong University of Science and Technology, Wuhan 430030, China; ${ }^{7}$ Department of Radiation-Oncology, First Hospital of Jilin University, Changchun I3002 I, China
\end{abstract}

*These authors contributed equally to this work

Correspondence: Shi-yin Xiao

Department of Neurosurgery, Second Affiliated Hospital of Guangzhou Medical University, 250 Changgang East Road, Haizhu, Guangzhou, Guangdong 510260, China

Tel+862087343310

Email xsy8II0@I63.com
Objective: Surgical resection serves an important role in the multidisciplinary treatment of cerebral metastases (CMs). Conventional white-light, microsurgical, and circumferential stripping of CMs is standard neurosurgical procedure, but is associated with a high recurrence rate. Based on this outcome, there is an urgent need for a new surgical strategy, such as fluorescenceguided resection, for CMs, in order to achieve total removal.

Methods: A retrospective study was carried out in 38 patients clinically and pathologically diagnosed with breast cancer brain metastasis at three medical centers from May 2012 to June 2016. The study comprised group 1 (fluorescein-guided surgery) and group 2 (standard microsurgery). In group $1,5 \mathrm{mg} / \mathrm{kg}$ of fluorescein sodium was injected intravenously after an allergy test and before general anesthesia for 17 patients. A yellow 560 filter was employed for microsurgical tumor resection. Group 2 consisted of 21 patients for whom fluorescein was not administered.

Results: Surgical outcomes were assessed concerning the extent of resection and Karnofsky performance status. Gross total resection was achieved in these patients, with high fluorescence markedly enhancing tumor visibility. The extent of resection had a powerful influence on performance status. Overall survival after CM was 24.1 months in patients given fluorescein and was 22.8 months in the nonfluorescein group.

Conclusion: Fluorescein-guided surgery is a simple, safe, and practical method to resect breast cancer brain metastasis, and leads to a higher proportion of resection compared to common microsurgery. This offers a tremendous advantage when navigating a tiny tumor, and improves the quality of life of patients with CM.

Keywords: fluorescein sodium, breast cancer brain metastasis, fluorescence-guided surgery, extent of resection, KPS

\section{Introduction}

Cerebral metastasis (CM) occurs in a high proportion of intracranial tumors. ${ }^{1,2}$ Many studies suggest that the morbidity of $\mathrm{CM}$ has been increasing. ${ }^{3} \mathrm{~A}$ large number of clinical studies have noted the value of total removal of CM for patients. ${ }^{4-6}$ The complete resection of $\mathrm{CM}$ has been shown to benefit patients and to be crucial for quality of life and the efficacy of subsequent treatments. ${ }^{7,8}$ Bright-light surgery (BLS) is usually insufficient in achieving the resection of $\mathrm{CM}$. The use of fluorescence-guided surgery (FGS) in brain tumors is a field of recent intense research. ${ }^{9}$ The use of FGS with fluorescein sodium (FL), used to stain tumors in patients with glioblastoma, malignant melanoma, and lymphoma, increases the resection rate. ${ }^{10}$ One issue in open surgery with the goal of complete removal of a tumor is how to illuminate the tumor 
precisely and where to stop resection to avoid postoperative functional deficits. Because contrast enhancement of tumor tissue is associated with the breakdown of the blood-brain barrier (BBB), these substances, such as FL, accumulate in metastatic tumor tissue through a disrupted BBB, which facilitates intraoperative visualization of tumor tissue. ${ }^{11-13}$ The present study reports the significance of FL-guided surgery in patients with breast cancer brain metastasis (BCBM).

\section{Methods}

\section{General patient data}

A retrospective study was carried out in 38 female patients from three medical centers who were clinically and pathologically diagnosed with BCBM from May 2012 to June 2016. The study comprised group 1 (FGS) and group 2 (BLS). Exclusion criteria in group one were patients under the age of 18 years, those who declined to participate, and patients with impaired liver or renal function or history of an allergic reaction against FL. Ages ranged between 32 and 73 years (mean age 55 years). Karnofsky performance status (KPS) was $\geq 60$ in all patients. The 17 patients who were approved to receive fluorescein administration constituted group 1 and were treated from 2014 to 2016, and group 2 consisted of 21 patients in whom fluorescein was not used from 2012 to 2014 (Table 1).

Informed consent was obtained from patients for the offlabel use of FL. All patients who wished to participate in the study were informed via standard procedures. Irrespective of extracranial disease status, there are indications for surgery in patients with symptomatic mass effect. The study was approved by the ethics committee of the Second Affiliated Hospital of Guangzhou Medical University. Comprehensive data, including preoperative and postoperative magnetic resonance imaging (MRI) scans within 72 hours, were

Table I Patient characteristics

\begin{tabular}{|l|l|l|}
\hline & Fluorescein & Nonfluorescein \\
\hline All & 17 & 21 \\
Extent of surgery & & \\
Gross total resection & $16(94 \%)$ & $13(62 \%)$ \\
KPStotal resection & $1(6 \%)$ & $8(38 \%)$ \\
immediately postoperative) & 75.72 & 76.42 \\
3 months postoperative & 80.00 & 73.33 \\
$\mathbf{6}$ months postoperative & 84.12 & 75.23 \\
I2 months postoperative & 82.94 & 74.76 \\
Chemotherapy & $17(100 \%)$ & 72.38 \\
Hormonotherapy & $11(65 \%)$ & $21(100 \%)$ \\
Brain radiotherapy & $1(6 \%)$ & $9(43 \%)$ \\
\hline
\end{tabular}

collected. A predefined dose of $5 \mathrm{mg} / \mathrm{kg}$ FL was injected intravenously via the central vein according to the scheduled steps. Neuronavigation was implied for craniotomy planning. FGS was performed with a surgical microscope (Pentero 900; Carl Zeiss, Oberkochen, Germany) equipped with a yellow 560 filter. Postoperatively, all patients were admitted to the neurosurgical common-care unit for postoperative care. Postoperative MR images were reviewed for any contrastenhancing residual tumor tissue by neuroradiologists and neurosurgeons. The diagnosis of CM (invasive ductal carcinoma) was confirmed in all patients by neuropathologists. Surgical outcome was divided into two groups: no residual tumor tissue $=$ GTR and residual tumor tissue $=$ subtotal resection (STR). Surgical records were screened for any reference to grade of fluorescent staining: "bright/helpful" vs "no fluorescence/not helpful".

\section{Radiological examination}

A total of 35 patients had one resectable CM each in the two groups, and another three patients had two CMs each. Our goal was to evaluate the extent of resection (EOR) and prognostic value under the resistance of FL. Postoperative multimodal MRI ( $T_{1}$-weighted, $T_{2}$-weighted, $T_{2}$ fluidattenuated inversion recovery, diffusion-weighted, enhanced $T_{1}$-weighted, and MR spectroscopy) was obtained within 72 hours for assessment of resection degree. GTR was defined as no residual enhanced tumors on postoperative gadoliniumenhanced $T_{1}$-weighted MRI and STR as the presence of residual tumor tissue.

\section{Surgical procedure}

FL ( $5 \mathrm{mg} / \mathrm{kg}$ body weight) was injected intravenously in group 1 after an allergy test and before the induction of anesthesia. A fluorescein-specific filter was employed for microsurgical tumor resection. Craniotomy was performed under neurosurgical navigation guidelines. Following administration of FL, labeled tumors were visualized with fluorescence microscopy. The surgical excision was based mainly on the identification of fluorescein-stained tumor tissue. Stained CMs were sufficiently bright to perform complete resection, and normal brain parenchyma was illuminated (Figure 1, A-D). The resection of all fluorescing tissues of a nonfunctional area did not cease, as tumor cells could not be excluded in intraoperative frozen sections. Postoperatively, the surgical resection cavity was imaged under both bright-field and fluorescent illumination to assess the degree of resection. In group 2, the standard 
microsurgical procedure was followed for resection of tumors. In most of these cases in the two groups, the tumor was removed en bloc, and in some cases it was removed piece by piece in conjunction with white light. When applicable, ultrasound was employed for tumor localization. No additional instruments or filters for the microscope were adopted. All patients were transferred to the oncology department after postoperative recovery.

\section{Compliance with ethical standards and informed consent}

All procedures performed in studies involving human participants were in accordance with the ethical standards of the institutional and/or national research committee and with the 1964 Helsinki Declaration and its later amendments or comparable ethical standards. Informed consent was obtained from all participants included in the study.

\section{Statistical analysis}

For comparison between the two groups, the extent of surgery (GTR and STR) by means of early postoperative contrastenhanced MRI between fluorescent and white-light assistance was analyzed via $\chi^{2}$ test and pre- and post-operative KPS score by the independent samples $t$-test. Statistical analysis of overall survival was performed using the Kaplan-Meier method and the significance in difference among survival curves calculated. Significance was defined as $P<0.05$.

\section{Results}

Surgical outcomes concerning fluorescence intensity, EOR, KPS score, and median survival were assessed. A total of 16 patients displayed bright fluorescent staining, which noticeably enhanced tumor visibility (Figure 1). For details, see Table 2. Insufficient FL staining was observed in one case. The presentation case displayed the impression of a fluorescent stained tumor below the cortex: the view under white light (Figure 1C) did not show an explicit lesion, but the view under the yellow filter (Figure 1D) depicted a fluorescence signal that clearly demarcated the targeted area. The final pathological diagnosis (invasive ductal carcinoma) in the two groups was confirmed.

GTR was achieved in 94\% (16 of 17) of patients in whom fluorescein was administered compared to patients

Table 2 Fluorescence staining and extent of resection (EOR)

\begin{tabular}{|l|l|l|l|}
\hline Age, years & Staining & Localization & EOR \\
\hline 49 & + & Right parietal & GTR \\
32 & + & Left occipital & GTR \\
67 & + & Right frontal & GTR \\
54 & + & Right temporal & GTR \\
49 & + & Left cerebellar & GTR \\
41 & + & Right parietal & GTR \\
55 & + & Right parietal & GTR \\
59 & + & Right parietal & GTR \\
62 & + & Right parietal & GTR \\
61 & + & Right parietal & GTR \\
67 & + & Left frontal & GTR \\
73 & + & Left frontal & GTR \\
39 & + & Left frontal & GTR \\
49 & - & Right frontal & STR \\
67 & + & Left occipital & GTR \\
68 & + & Right temporal & GTR \\
47 & + & Left frontal & GTR \\
\hline
\end{tabular}

Notes: +, bright and marked fluorescence; -, no or insufficient fluorescence. Abbreviations: GTR, gross total resection; STR, subtotal resection.

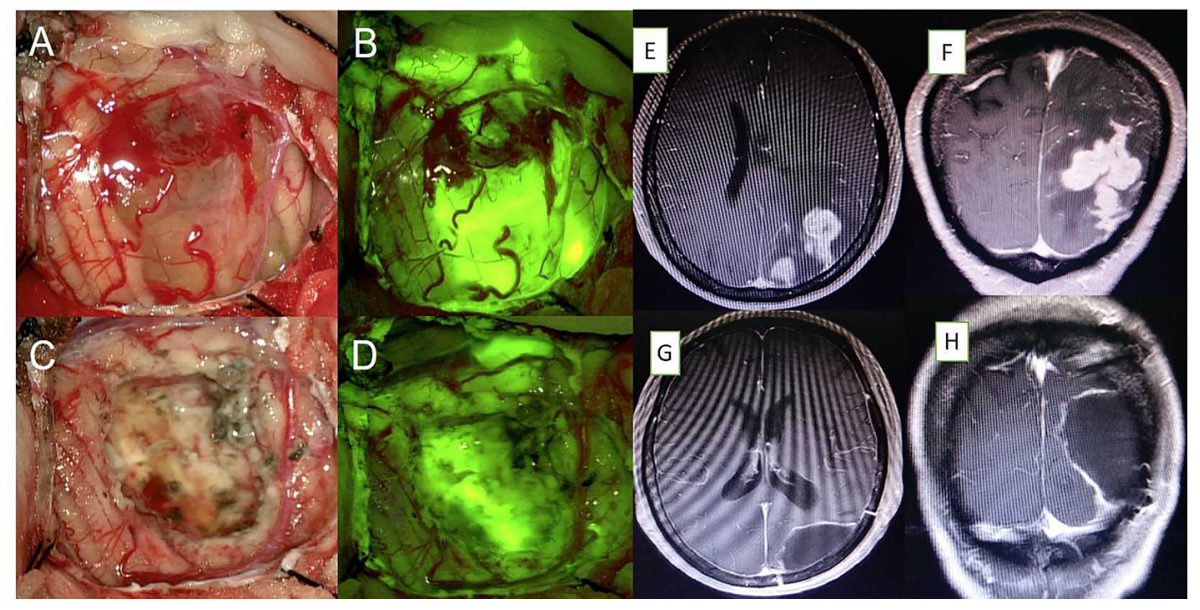

Figure I (A) Microsurgical photograph of the right parietal lobe under white light and (B) the yellow 560 filter from initial stages of tumor resection. (C, D) Residual tissue located in the deep area of the resection cavity was identified under fluorescence compared to white light. (E-H) Axial and sagittal magnetic resonance imaging showing complete removal of the tumor. 
who underwent BLS $(62 \%, 13$ of 21 ; Table 1$)$. No patients with brightly fluorescent stained tumors in the FGS group showed significant residual tumor tissues on postoperative MRI examination (Figure 1, E-H). One case was classified as STR due to pale FL staining. In group 2, a radiographic examination could not exclude the possibility of residual tumor tissues. These tumors were classified as STR. STR was obtained in eight of 21 patients. There was a significant difference in GTR rate between the two groups $(P=0.02)$. Median survival was 22.8 months in the nonfluorescein group and 24.1 months in the FL group. There was no significant difference in survival between the two groups (Figure 2). However, EOR had a powerful influence on patients' KPS scores 6-12 months after surgery. Patients' KPS scores in the FGS group were higher than in the BLS group, who received radiotherapy 3 months later (Table 1, Figure 3).

The average duration of the surgical procedure (skin to skin) in group 1 was slightly shorter than in group 2 . The median duration of the in-hospital stay was almost identical in the two groups. Transient neurological deterioration was documented in three patients, and a new postoperative permanent neurological deficit was present in one patient. Patients with complete resection did not have adjuvant radiation therapy for the brain. No adverse events or complications related to FL were observed during the postoperative course.

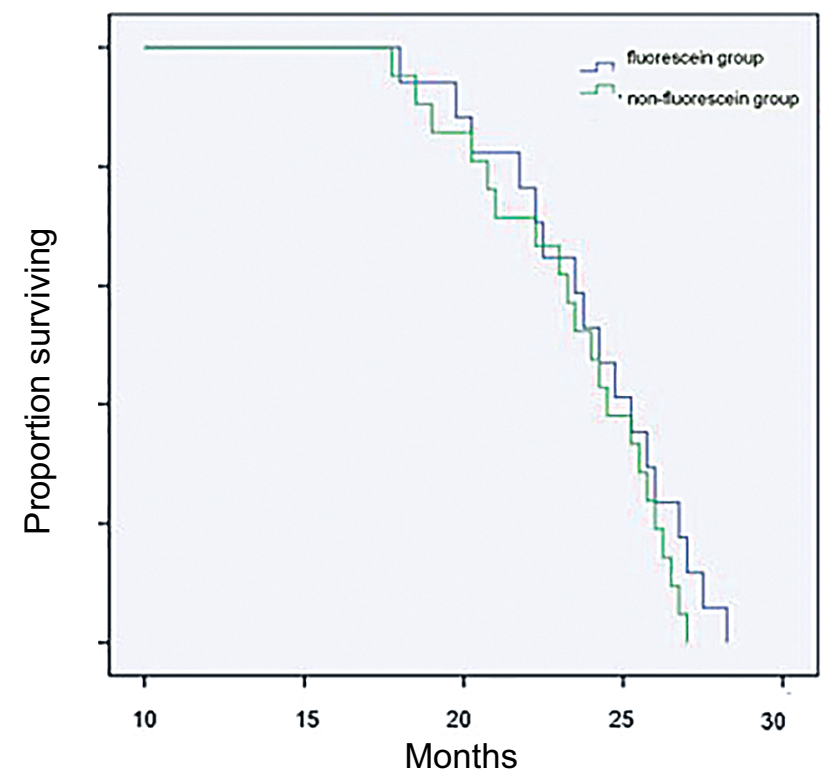

Figure 2 Kaplan-Meier survival curve: correspondence of fluorescein sodium group ( 17 patients) and with nonfluorescein sodium (2I patients) group.

\section{Discussion}

An increasing prevalence of CMs, which outnumber primary brain tumors, has appeared in adults. ${ }^{14}$ In brain-metastasis surgery, it is still difficult to identify resection margins, even with intraoperative ultrasound or neuronavigation. ${ }^{15,16}$ Accurate location and complete excavation of lesions play a crucial role in the multidisciplinary treatment of CMs. The detection of macroscopic CM lesions is largely limited by the weak contrast between surrounding normal tissue and tumor lesions. FGS has been documented to promote the resection of malignant gliomas. ${ }^{13,17,18}$ For BCBMs, FGS has not been reported in the published literature.

With the development of surgical tools, the successful surgical resection rate has risen., ${ }^{3,19}$ Modern neurosurgical tools have facilitated craniotomy and tumor resection enormously. The accurate orientation and differentiation of a tumor from normal brain tissue is an intractable issue. ${ }^{20} \mathrm{~A}$ kind of fluorescent tracer has been investigated for the intraoperative detection of tumor tissues. ${ }^{13}$ FGS resection of some types of CMs has been confirmed to be an effective and safe technique that improves the EOR. ${ }^{21}$ We evaluated FGS values in the present series of patients with BCBM. FL permeated the damaged $\mathrm{BBB}$ in tumor tissue and likewise passively accumulated within the disrupted BBB, which indicates strong accordance between intraoperative fluorescence and preoperative radiographic enhancement. A color change from white to yellow fluorescence clearly indicated the presence of a tumor lesion after intravenous injection of FL. The labeled tumors could then be removed under fluorescence guidance. This technique minimized normal-tissue manipulation and facilitated the microsurgical dissection of the lesion from the surrounding parenchyma.

In this retrospective study, we compared the influence of FGS on resection extension in two groups of patients with CMs. Surgical adjuncts, such as intraoperative ultrasound and navigation, can aid in surgical decisions to some degree, but they cannot make the tumor margin invisible. ${ }^{19}$ Research has indicated that FL makes the surgery easier and results in a higher resection rate compared to patients in whom fluorescein is not used. ${ }^{13} \mathrm{FL}$ fluoresces metastasis, which can be visualized in the setting of a normal-appearing background and is sufficiently strong to perform complete resection so that intraoperative fluorescence can help identify the target area.

FGS is an easy procedure, and does not require additional time or instrumentation. The intraoperative application of fluorescence is also a convenient, easily accessible manipulation that fixes the mass precisely and preliminarily aids in the assessment of the tumor-resection rate via fluorescence-signal 


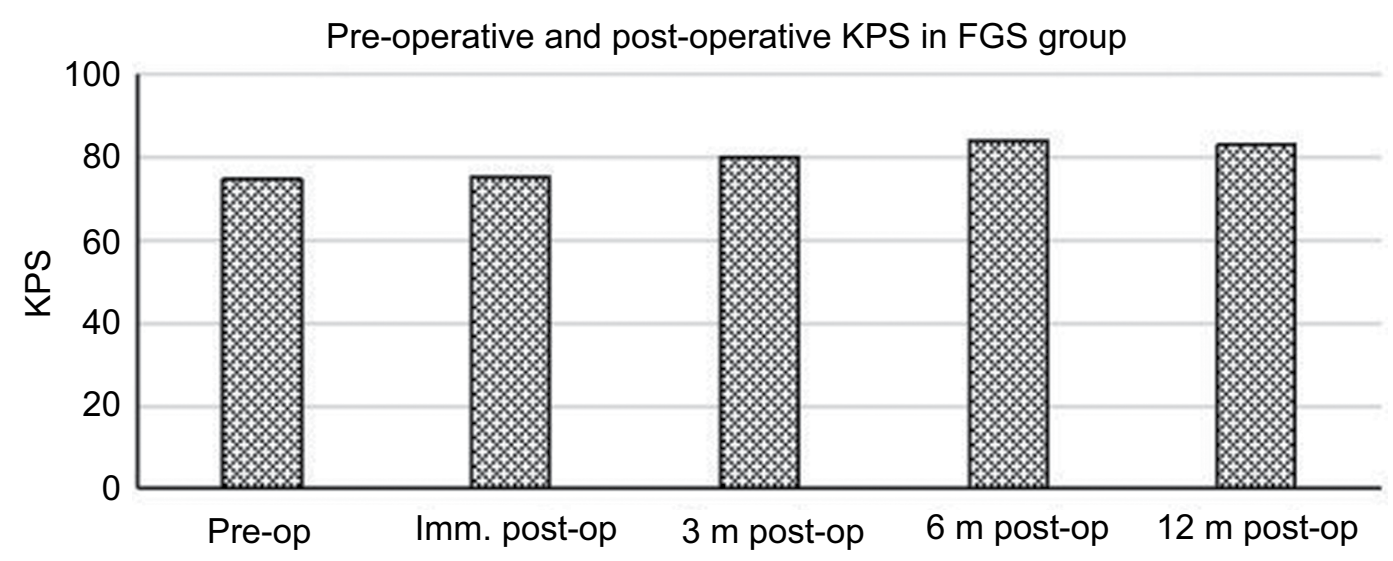

Pre-operative and post-operative KPS in BLS group

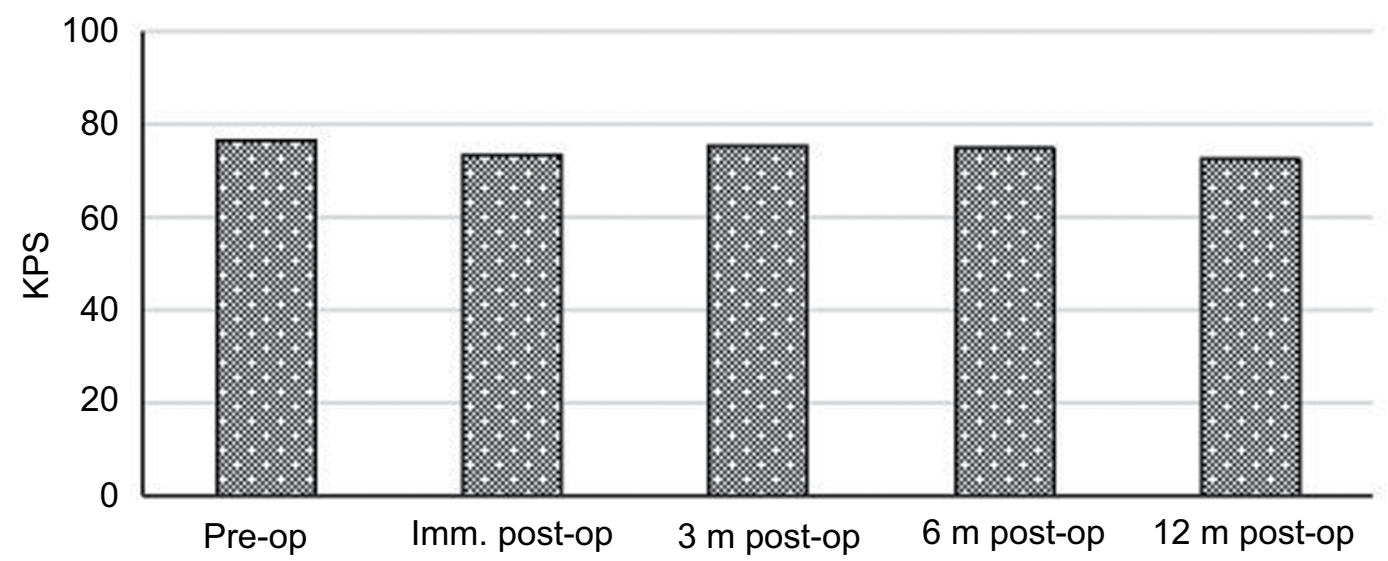

Figure 3 Preoperative, immediate (imm), and 3-, 6-, and 12-month postoperative Karnofsky performance status (KPS). Abbreviations: BLS, bright-light surgery; FGS, fluorescence-guided surgery.

variation. During the course of removal, the microscope can be switched alternatively from fluorescent to white-light illumination as desired by the neurosurgeon. Utilizing the microscope with a yellow 560 filter, the identification of residual tumor tissues in the surgical bed facilitated complete resection, which was verified on postoperative MRI. The study had been performed in a glioma-outcome study. ${ }^{22}$ Nontumor-specific fluorescence gathered at metastatic tumors, which were dyed yellow under fluorescence microscopy. In our continuous series, the data suggest that the use of FL led to a higher proportion of resection of BCBM than conventional microsurgery. Macroscopically, we observed that fluorescence appeared in the tumor bed during surgery, but the fluorescence of normal brain tissue was light, and the texture and color of yellow-stained tissue were different from these metastatic tumors. The following histopathological tests confirmed our intraoperative judgment.

FL has been increasingly reported to improve the efficacy of fluorescence-guided resection of malignant gliomas. ${ }^{23-25}$
FL gathers in a disrupted-BBB area as a result of pathologically increased vascular permeability in the adjacent tissue of metastatic cancers. ${ }^{26,27}$ EOR was evaluated by means of early postoperative contrast-enhanced MRI. The GTR rate in group 1 was noticeably higher than in group 2 . By the use of a dedicated filter on the surgical microscope, it was relatively easy to visualize discretely fluorescent tumors after injection of a low dose of FL. The visible fluorescence effect strongly confirmed the accurate area of the tumors and decreased surgical damage to normal brain tissue via dissection of the edema zone. A bright-fluorescence signal was achieved in most patients in group 1, which aided in identification of suspicious lesions. Accordingly, application of FL in the BCBMs confirmed the location of the surgical site and resulted in safe and undetectable residual resection. Our findings are similar to data provided by Schebesch et al. ${ }^{28}$ In the current study, our analysis demonstrated a positive fluorescein-staining rate of $94 \%$ and just one case with insufficient tumor fluorescence staining in group 1 . To the best of 
our knowledge, our series of patients provide the first data on the efficacy of FL in patients with BCBMs. The intraoperative use of FL aided in surgical decisions where the tumor border was clearly distinguishable from surrounding brain tissue and improved the complete resection of BCBM. The content of this study surpassed that of a simple feasibility study in patients with BCBM. Despite the limited number of patients, this study illustrated that FL facilitated complete removal of these metastatic tumors.

Complete resection of contrast-enhanced CM has been repeatedly indicated to be crucial for decreasing the risk of recurrence and increasing the length and quality of survival in CM. ${ }^{29,30}$ Although FGS failed to affect OS in the series, patients with complete tumor resection had higher KPS scores. Patients were clinically examined by assessing KPS scores and eventual appearance of new neurological deficits or worsening of preoperative deficits within 72 hours after surgery, and then during follow-up visits within 1 year. KPS scores in the FGS group declined slightly in the immediate postoperative period and then returned to baseline level $<3$ months postoperatively, similar to what was observed in the BLS group. However, from 3 months to 1 year after surgery, KPS scores of patients with complete tumor resection gradually ameliorated, and with incomplete tumor removal receiving radiotherapy for residual metastasis, scores slowly decreased. The relationship between EOR, KPS score, and subsequent radiotherapy for residual tumors is of practical value for patients with brain metastasis from breast cancer. Radiotherapy for residual metastasis reduces the relapse rate at the initial site, but it also causes side effects, such as a delayed irreversible decline in radionecrosis and radiationinduced neurocognitive impairment. ${ }^{31,32}$

The limited number of patients and other parameters that affected survival may explain the lack of statistical significance for median survival time. We are well aware of the limitations of our study: it was retrospective, and no qualitative statements about fluorescent staining in patients with $\mathrm{BCBM}$ can be made. In terms of surgical time, the average time course of the FGS procedure for BCBM was slightly shorter than in the standard-microsurgery group. None of the patients complained about any systemic or local side effects. No adverse effects or anaphylactic disorders were observed, so FL was shown to be safe in patients with BCBMs.

\section{Limitations}

This study has some limitations. It was retrospective study of a relatively small sample; therefore, a randomized controlled trial with a larger sample is warranted to explore the effects of FL-guided resection of BCBM over standard resection techniques.

\section{Conclusion}

Our preliminary data revealed the potential benefit of FLguided resection of $\mathrm{BCBM}$ over standard resection techniques. An improvement in intraoperative detection of $\mathrm{CM}$ lesions with FL would essentially facilitate complete resection. FGS is a simple, safe, and practical method of resecting BCBM, which results in superior resection compared to BLS, lowers the probability of subsequent radiation therapy for $\mathrm{CM}$, improves the quality of life of patients with $\mathrm{CM}$, and reduces medical costs. However, these effects should be confirmed in a randomized controlled clinical trial.

\section{Acknowledgments}

Relevant human materials were reviewed and approved by the ethics committee of the Second Affiliated Hospital of Guangzhou Medical University, Sun Yat-sen University Cancer Center and Affiliated Tumor Hospital of Xinjiang Medical University, and the study was deemed to meet the medical ethical requirements. We wish to thank the patients and families involved in this study for providing written informed consent for publication of the accompanying images. This work was supported by the Science and Technology Planning Project of Guangdong Province, China (2012B031800102), National Natural Science Foundation of China (grant 81500830), Science and Technology Program of Guangzhou, China (grant 201607010365) and High Level University Construction Project of Guangzhou University of Traditional Chinese Medicine (grant A1-AFD018171Z11072).

\section{Disclosure}

The authors report no conflicts of interest in this work.

\section{References}

1. Alexandru D, Bota DA, Linskey ME. Epidemiology of central nervous system metastases. Prog Neurol Surg. 2012;25:13-29.

2. Stark AM, Tscheslog H, Buhl R, Held-Feindt J, Mehdorn HM. Surgical treatment for brain metastases: prognostic factors and survival in 177 patients. Neurosurg Rev. 2005;28(2):115-119.

3. Nieder C1, Spanne O, Mehta MP, Grosu AL, Geinitz H. Presentation, patterns of care. and survival in patients with brain metastases: what has changed in the last 20 years? Cancer. 2011;117:2505-2512.

4. Gates M, Alsaidi M, Kalkanis S. Surgical treatment of solitary brain metastases. Prog Neurol Surg. 2012;25:74-81.

5. Lee CH, Kim DG, Kim JW, et al. The role of surgical resection in the management of brain metastasis: a 17-year longitudinal study. Acta Neurochir. 2013;155(3):389-397.

6. Patchell RA, Tibbs PA, Walsh JW, et al. A randomized trial of surgery in the treatment of single metastases to the brain. $N$ Engl $J$ Med. 1990;322(8):494-500. 
7. Kamp MA, Dibué M, Niemann L, et al. Proof of principle: supramarginal resection of cerebral metastases in eloquent brain areas. Acta Neurochir. 2012;154(11):1981-1986.

8. Okuda T, Kataoka K, Yabuuchi T, Yugami H, Kato A. Fluorescenceguided surgery of metastatic brain tumors using fluorescein sodium. J Clin Neurosci. 2010;17(1):118-121.

9. Bouvet M, Hoffman RM. Glowing tumors make for better detection and resection. Sci Transl Med. 2011;3(110):fs10.

10. Schebesch KM, Hoehne J, Hohenberger C, et al. Fluorescein sodiumguided surgery in cerebral lymphoma. Clin Neurol Neurosurg. 2015;139: 125-128.

11. Schneider SW, Ludwig T, Tatenhorst L, et al. Glioblastoma cells release factors that disrupt blood-brain barrier features. Acta Neuropathol. 2004;107(3):272-276.

12. Shinoda J, Yano H, Yoshimura S, et al. Fluorescence-guided resection of glioblastoma multiforme by using high-dose fluorescein sodium. Technical note. J Neurosurg. 2003;99(3):597-603.

13. Stummer W, Pichlmeier U, Meinel T, Wiestler OD, Zanella F, Reulen HJALA-Glioma Study Group. Fluorescence-guided surgery with 5-aminolevulinic acid for resection of malignant glioma: a randomised controlled multicentre phase III trial. Lancet Oncol. 2006;7(5):392-401.

14. Sawaya R, Ligon BL, Bindal RK. Management of metastatic brain tumors. Ann Surg Oncol. 1994;1(2):169-178.

15. Cheon JE. Intraoperative neurosonography revisited: effective neuronavigation in pediatric neurosurgery. Ultrasonography. 2015;34(2):79-87.

16. Kuhnt D, Bauer MH, Nimsky C. Brain shift compensation and neurosurgical image fusion using intraoperative MRI: current status and future challenges. Crit Rev Biomed Eng. 2012;40(3):175-185.

17. Pichlmeier U, Bink A, Schackert G, Stummer WA, LA Glioma Study Group. Resection and survival in glioblastoma multiforme: an RTOG recursive partitioning analysis of ALA study patients. Neuro Oncol. 2008;10(6):1025-1034.

18. Acerbi F, Broggi M, Schebesch KM, et al. Fluorescein-Guided Surgery for Resection of High-Grade Gliomas: A Multicentric Prospective Phase II Study (FLUOGLIO). Clin Cancer Res. 2018;24(1):52-61.

19. Wen PY, Loeffler JS. Brain metastases. Curr Treat Options Oncol. 2000;1(5):447-457.

20. Höhne J, Hohenberger C, Proescholdt M, et al. Fluorescein sodiumguided resection of cerebral metastases-an update. Acta Neurochir. 2017;159(2):363-367.
21. Okuda T, Kataoka K, Yabuuchi T, Yugami H, Kato A. Fluorescenceguided surgery of metastatic brain tumors using fluorescein sodium. J Clin Neurosci. 2010;17(1):118-121.

22. Stummer W, Novotny A, Stepp H, Goetz C, Bise K, Reulen HJ. Fluorescence-guided resection of glioblastoma multiforme by using 5-aminolevulinic acid-induced porphyrins: a prospective study in 52 consecutive patients. J Neurosurg. 2000;93(6):1003-1013.

23. Acerbi F, Broggi M, Eoli M, et al. Is fluorescein-guided technique able to help in resection of high-grade gliomas? Neurosurg Focus 2014;36(2):E5.

24. LiY, Rey-Dios R, Roberts DW, Valdés PA, Cohen-Gadol AA. Intraoperative fluorescence-guided resection of high-grade gliomas: a comparison of the present techniques and evolution of future strategies. World Neurosurg. 2014;82(1-2):175-185.

25. Rey-Dios R, Cohen-Gadol AA. Technical principles and neurosurgical applications of fluorescein fluorescence using a microscopeintegrated fluorescence module. Acta Neurochir. 2013;155(4): 701-706.

26. Lee J, Baird A, Eliceiri BP. In vivo measurement of glioma-induced vascular permeability. Methods Mol Biol. 2011;763:417-422.

27. Nduom EK, Yang C, Merrill MJ, Zhuang Z, Lonser RR. Characterization of the blood-brain barrier of metastatic and primary malignant neoplasms. J Neurosurg. 2013;119(2):427-433.

28. Schebesch KM, Hoehne J, Hohenberger C, et al. Fluorescein sodiumguided resection of cerebral metastases - experience with the first 30 patients. Acta Neurochir. 2015;157(6):899-904.

29. Kamp MA, Dibue M, Santacroce A, et al. The tumour is not enough or is it? Problems and new concepts in the surgery of cerebral metastases. Cancer. 2013;7:305-312.

30. Yoo H, Kim YZ, Nam BH, et al. Reduced local recurrence of a single brain metastasis through microscopic total resection. J Neurosurg. 2009;110(4):730-736.

31. Kocher M, Soffietti R, Abacioglu U, et al. Adjuvant whole-brain radiotherapy versus observation after radiosurgery or surgical resection of one to three cerebral metastases: results of the EORTC 22952-26001 study. J Clin Oncol. 2011;29(2):134-141.

32. Tallet AV, Azria D, Barlesi F, et al. Neurocognitive function impairment after whole brain radiotherapy for brain metastases: actual assessment Radiat Oncol. 2012;7(1):77-78.
Cancer Management and Research

\section{Publish your work in this journal}

Cancer Management and Research is an international, peer-reviewed open access journal focusing on cancer research and the optimal use of preventative and integrated treatment interventions to achieve improved outcomes, enhanced survival and quality of life for the cancer patient. The manuscript management system is completely online and includes

\section{Dovepress}

a very quick and fair peer-review system, which is all easy to use. Visit http://www.dovepress.com/testimonials.php to read real quotes from published authors. 